Supplementary Figure 1, Heat map illustrating the relative ratio of the identified metabolites during the validation of quenching of metabolism. The chemostat was operated under the first glucose limitation $(2 \mathrm{~g} \mathrm{~L}-1)$ and each value represents the averaged response of five independent samples. The response of the metabolites was split into four sections, 0.001- 0.0099, 0.01-0.099, 0.1-0.99, 1- 9.99 as illustrated by the increasing grey scale.

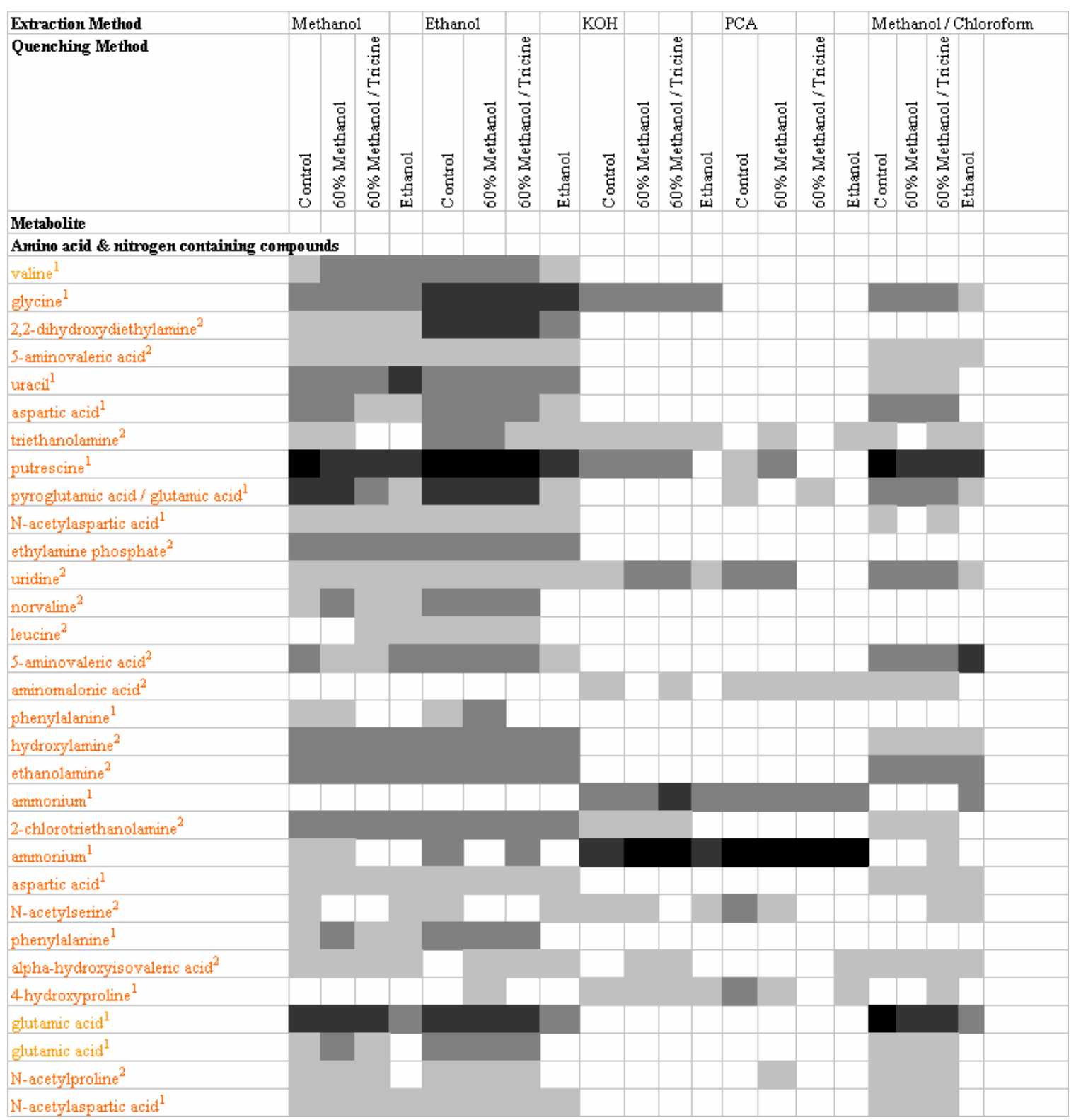


Continued Supplementary Figure 1

\begin{tabular}{|l|}
\hline Extraction Method \\
\hline Quenching Method \\
\hline
\end{tabular}

Methanol

\section{Fatty acids}
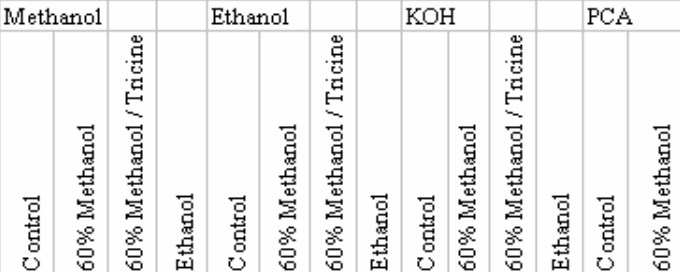

dodecanoic acid ${ }^{2}$

myristic acid ${ }^{1}$

pentadecanoic acid ${ }^{2}$

hexadecanoic acid ${ }^{1}$

'hexadecenoic acid ${ }^{1}$

1-octadecanol ${ }^{2}$

heptadecanoic acid ${ }^{2}$

octadecanoic acid

oleic acid ${ }^{1}$

eicosanoic acid ${ }^{1}$

dehydroabietic acid ${ }^{2}$

palmitin $^{1}$

9-tetradecenoic acid ${ }^{2}$

heptadecanoic acid ${ }^{2}$

pentadecanoic acid ${ }^{2}$

9-tetradecenoic acid $^{2}$

'fatty acid ${ }^{2}$

'fatty acid ${ }^{2}$

2-monopalmitin ${ }^{2}$

nonanoic acid ${ }^{2}$

2-monostearin ${ }^{2}$

monostearin ${ }^{2}$

Organic acids

mercaptoacetic acid ${ }^{2}$

1,2,3-trihydroxybenzoic acid ${ }^{2}$

benzoic acid derivative ${ }^{2}$

4hydroxybenzoic acid ${ }^{2}$

citric acid ${ }^{1}$

4hydroxybutyric acid ${ }^{1}$

phosphoric acid, monomethyl ester ${ }^{2}$

octanoic acid ${ }^{1}$

decanoic acid ${ }^{1}$

2-hydroxyglutaric acid ${ }^{1}$

threonic acid ${ }^{1}$

fumaric acid ${ }^{1}$

lactic acid ${ }^{1}$

lactic acid ${ }^{1}$

pyruvic acid ${ }^{1}$

citramalic acid ${ }^{1}$

acetic acid ${ }^{2}$

2,4-dihydroxybutanoic acid ${ }^{2}$

succinic acid ${ }^{1}$

formic acid ${ }^{2}$

succinic acid ${ }^{1}$

2,4,5-trihydroxypentanoic acid ${ }^{2}$

2-methyl, 4hydroxybutyric acid ${ }^{2}$

2-isopropylmalic acid ${ }^{1}$

2-hydroxysebacic acid ${ }^{2}$

4 hydroxyphenyllactic acid ${ }^{2}$

levulinic acid ${ }^{2}$
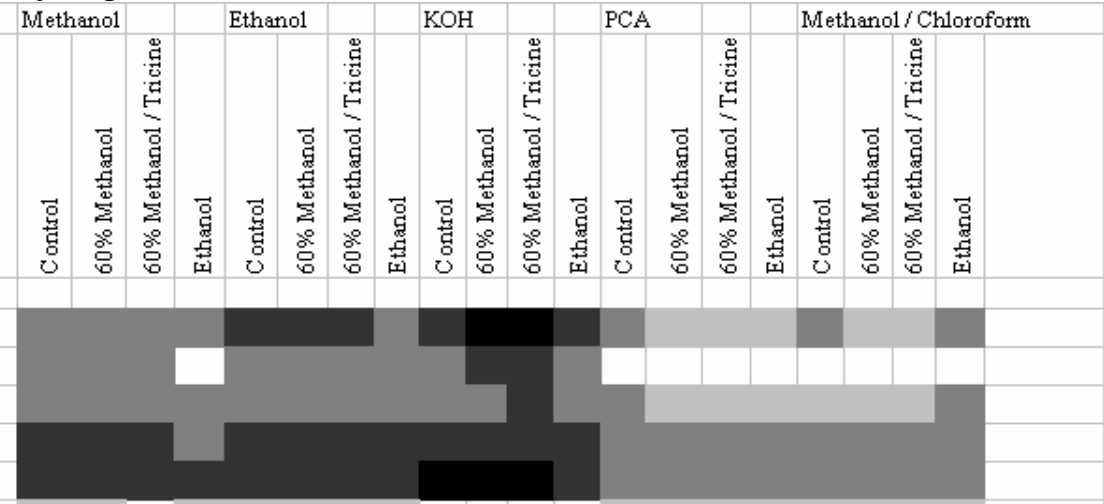


\section{Continued Supplementary Figure 1}

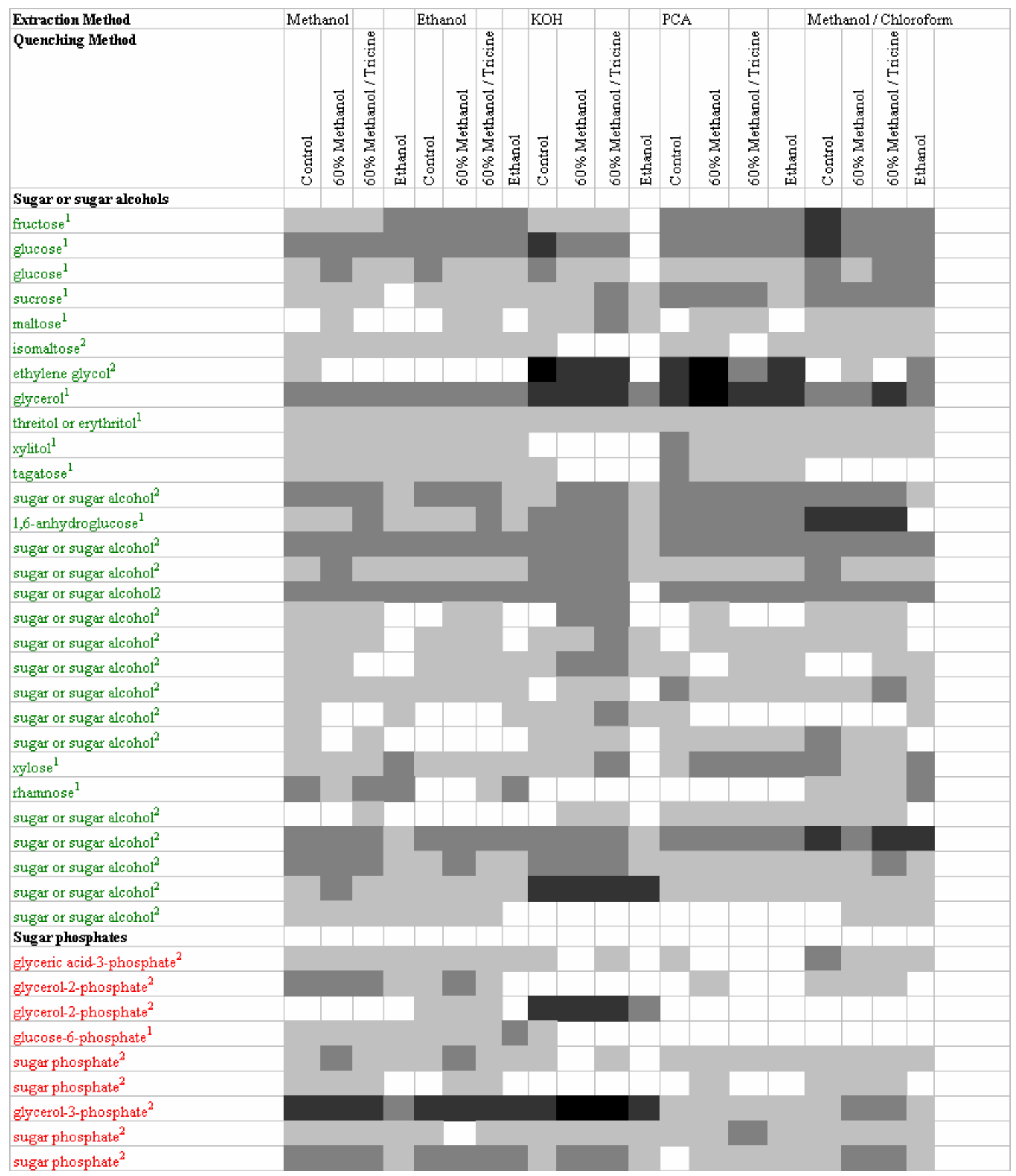




\section{Continued Supplementary Figure 1}

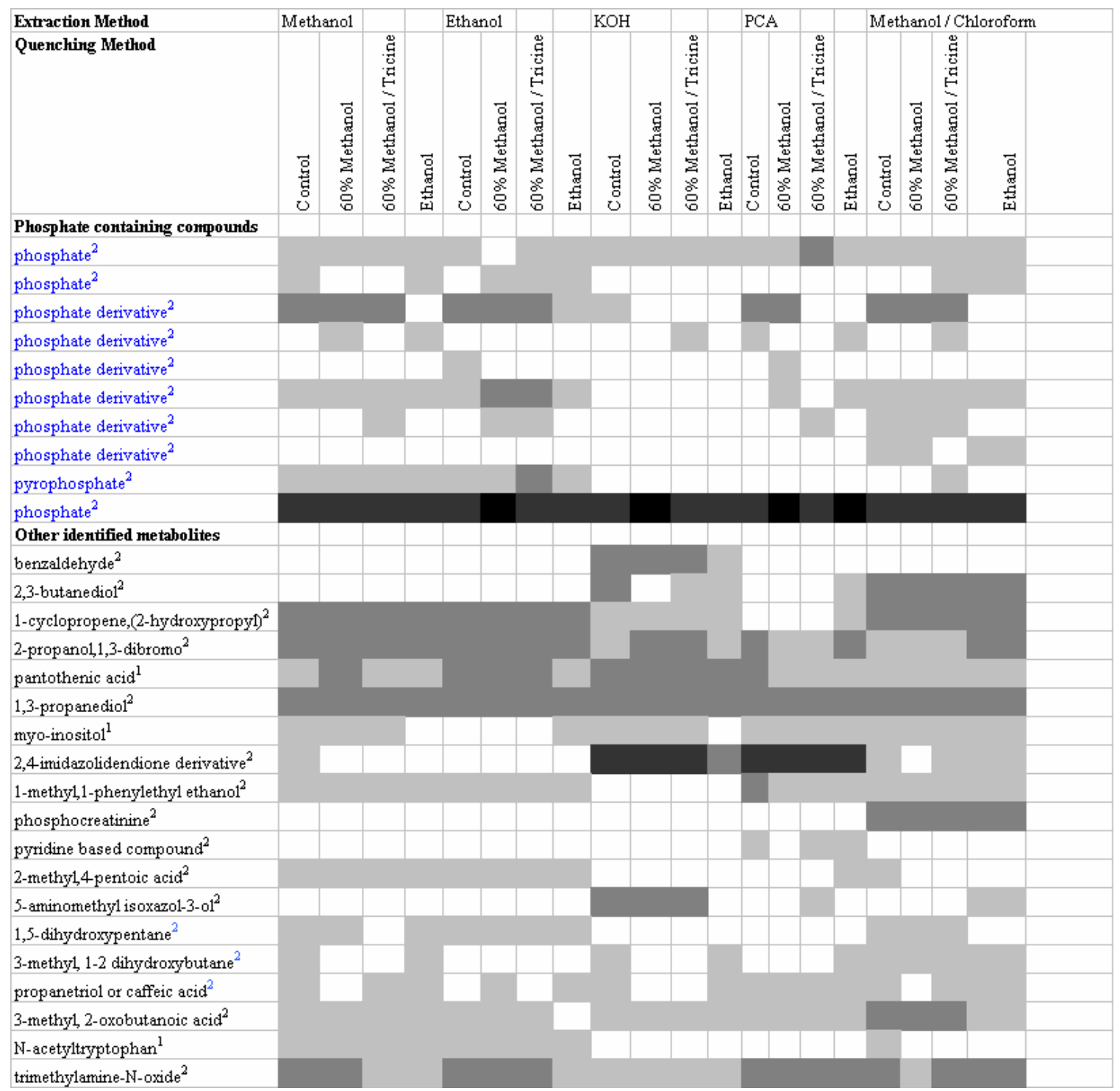


Supplementary Table 1, Identification of metabolites from the quenching supernatants and footprint samples illustrates in the Venn diagram (Figure 3). The columns (A-M) indicate the different sections illustrated in the Venn diagram. The figures indicate the number of peaks identified as a particular metabolite in that section. ('definative identification, matched to authentic standard by retention index and mass spectrum, ${ }^{2}$ identified by mass spectrum only).

\begin{tabular}{|c|c|c|c|}
\hline A & Cont' A & $\mathrm{D}$ & I \\
\hline $\begin{array}{l}\text { 1,2,3-trihydroxybenzoic } \\
\text { acid }^{2}\end{array}$ & nonanoic acid $^{2}$ & lactic acid ${ }^{1}$ & unknown peaks (4) \\
\hline 1,3-propanediol ${ }^{1}$ & octadecanoic acid $^{1}$ & sugar phosphate $^{2}$ & $\mathrm{~J}$ \\
\hline 1,5-dihydroxypentane ${ }^{2}$ & 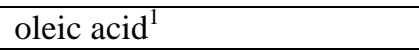 & tagatose $^{1}$ & octanoic acid $^{1}$ \\
\hline 1,6-anhydroglucose ${ }^{1}$ & palmitin $^{1}$ & phenylalanine $^{1}$ & unknown peaks (2) \\
\hline $\begin{array}{l}\text { 1-cyclopropene(2- } \\
\text { hydroxypropyl) }\end{array}$ & pantothenic acid ${ }^{\top}$ & unknown peaks (2) & $\mathrm{K}$ \\
\hline $\begin{array}{l}\text { 1-methyl,1-phenylethyl } \\
\text { ethanol }^{2}\end{array}$ & pentadecanoic $\operatorname{acid}^{2}(2)$ & $\mathrm{E}$ & myo-inositol $^{1}$ \\
\hline 1-octadecanol ${ }^{1}$ & phosphate $^{1}$ & maltose $^{1}$ & unknown peaks (6) \\
\hline 2,3-butanediol ${ }^{1}$ & propanetriol or caffeic acid ${ }^{2}$ & 2-monopalmitin ${ }^{2}$ & $\mathrm{~L}$ \\
\hline $\begin{array}{l}\text { 2,4-imidazolidendione } \\
\text { derivative }^{2}\end{array}$ & pyruvic acid ${ }^{l}$ & sugar or sugar alcohol ${ }^{2}$ & sugar or sugar alcohol ${ }^{2}$ \\
\hline 2-hydroxyglutaric acid ${ }^{1}$ & 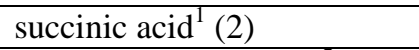 & myristic acid $^{1}$ & glycerol-2-phosphate $^{2}$ \\
\hline 2 -isopropylmalic acid ${ }^{1}$ & sugar or sugar alcohol ${ }^{2}(10)$ & unknown peak & unknown peaks (2) \\
\hline $\begin{array}{l}\text { 2-methyl, 4-hydroxybutyric } \\
\text { acid }^{2}\end{array}$ & $\mathrm{~B}$ & $\mathrm{~F}$ & $\mathrm{M}$ \\
\hline $\begin{array}{l}\text { 3-methyl, 2-oxobutanoic } \\
\text { acid }^{2}\end{array}$ & 2-hydroxysebacic acid ${ }^{2}$ & $\begin{array}{l}\text { 5-aminomethyl isoxazol-3- } \\
\mathrm{ol}^{2}\end{array}$ & phosphate $^{1}$ \\
\hline 4-hydroxybenzoic acid (3) & 2-methyl, 4-pentoic acid ${ }^{2}$ & $\begin{array}{l}\text { phosphoric acid, } \\
\text { monomethyl ester }\end{array}$ & 4-hydroxyphenyllactic acid ${ }^{2}$ \\
\hline 9-tetradecenoic acid ${ }^{2}$ & 2-propanol,1,3-dibromo ${ }^{2}$ & 2-chlorotriethanolamine ${ }^{2}$ & sugar or sugar alcohol ${ }^{2}$ \\
\hline 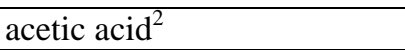 & 4-hydroxyproline ${ }^{1}$ & sucrose $^{1}$ & unknown peaks (2) \\
\hline $\begin{array}{l}\text { alpha-hydroxyisovaleric } \\
\text { acid }^{2}\end{array}$ & 5 -aminovaleric acid $^{2}(2)$ & formic acid ${ }^{2}$ & \\
\hline ammonium $^{1}(2)$ & ${\text { aspartic } \text { acid }^{1}(2)}$ & unknown peak & \\
\hline 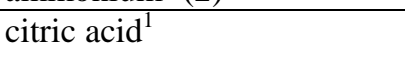 & citramalic acid $^{1}$ & $\mathrm{G}$ & \\
\hline dehydroabietic acid $^{2}$ & ethanolamine $^{2}$ & uracil $^{1}(2)$ & \\
\hline dodecanoic acid ${ }^{2}$ & 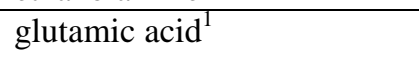 & 2-monostearin ${ }^{2}$ & \\
\hline fructose $^{1}$ & glycine $^{1}$ & ethylene glycol $^{2}$ & \\
\hline glucose $^{1}(2)$ & $\mathrm{N}$-acetylproline ${ }^{2}$ & ethylamine phosphate $^{2}$ & \\
\hline glyceric acid-3-phosphate $^{2}$ & N-acetyltryptophan ${ }^{1}$ & unknown peaks (2) & \\
\hline glycerol $^{1}$ & putrescine $^{1}$ & $\mathrm{H}$ & \\
\hline glycerol-3-phosphate ${ }^{2}$ & Unknown peaks (13) & phosphate $^{1}$ & \\
\hline heptadecanoic acid ${ }^{2}$ (3) & $\mathrm{C}$ & fumaric acid $^{1}$ & \\
\hline isomaltose $^{2}$ & phosphate $^{1}$ & norvaline $^{2}$ & \\
\hline lactic acid ${ }^{1}$ & (benzene based) $^{2}$ & glucose-6-phosphate $^{1}$ & \\
\hline monostearin $^{2}$ & Unknown peaks (2) & $\begin{array}{l}\text { pyroglutamic acid / } \\
\text { glutamic acid }^{1}\end{array}$ & \\
\hline $\mathrm{N}$-acetylaspartic acid ${ }^{1}$ & & unknown peaks (4) & \\
\hline $\mathrm{N}$-acetylserine ${ }^{2}$ & & & \\
\hline
\end{tabular}


Supplementary Table 2, Identification of metabolites from the different extraction methods illustrated in the Venn diagram (Figure 5). The columns (A-M) indicate the different sections illustrated in the Venn diagram. The figures in brackets indicate the number of peaks identified as a particular metabolite in that section and metabolites in bold indicate a particular peak not included in the Venn diagram due to constraints in the figure. ( ${ }^{1}$ definative identification, matched to authentic standard by retention index and mass spectrum, ${ }^{2}$ identified by mass spectrum only).

\begin{tabular}{|c|c|c|}
\hline A & $\mathrm{F}$ & Continued J \\
\hline phosphate derivative & 2,2-dihydroxydiethylamine ${ }^{2}$ & phosphate $^{1}$ \\
\hline \multirow[t]{2}{*}{ unknown peaks $(2+\mathbf{2})$} & dehydroabietic acid ${ }^{2}$ & pyrophosphate $^{2}$ \\
\hline & ethylamine phosphate ${ }^{2}$ & rhamnose $^{1}$ \\
\hline B & leucine $^{1}$ & sugar or sugar alcohol ${ }^{2}$ \\
\hline 4-hydroxyphenyllactic acid ${ }^{2}$ & norvaline $^{2}$ & unknown peaks (14) \\
\hline sugar phosphate ${ }^{2}$ & phenylalanine $^{1}(2)$ & \\
\hline unknown peak & valine $^{1}$ & $\mathrm{~K}$ \\
\hline 4-hydroxyphenyllactic acid ${ }^{2}$ & unknown peaks $(5+\mathbf{1})$ & unknown peaks (4) \\
\hline $\bar{C}$ & $\mathrm{G}$ & $\mathrm{L}$ \\
\hline decanoic acid $^{1}$ & glycerol-2-phosphate $^{2}$ & unknown peaks $(2+\mathbf{1})$ \\
\hline lactic acid ${ }^{1}$ & unknown peaks $(1+\mathbf{1})$ & \\
\hline octanoic acid $^{1}$ & & $\mathrm{M}$ \\
\hline phosphate derivative $^{2}$ & $\mathrm{H}$ & 2-propenoic acid, 3-methoxy 2-hydroxy ${ }^{2}$ \\
\hline Phosphocreatinine & glucose-6-phosphate $^{1}$ & \\
\hline \multirow[t]{2}{*}{ unknown peaks $(3+2)$} & ${\text { myristic } \text { acid }^{1}}^{2}$ & $\mathrm{~N}$ \\
\hline & unknown peaks (3) & 1-methyl,1-phenylethyl ethanol ${ }^{2}$ \\
\hline $\mathrm{D}$ & & 1-octadecanol ${ }^{1}$ \\
\hline (benzene based) $^{2}$ & I & 2-methyl, 4-pentoic acid2 \\
\hline phosphate derivative $^{2}$ & 2-hydroxysebacic acid ${ }^{2}$ & 2-monopalmitin ${ }^{2}$ \\
\hline pyridine based compound ${ }^{2}$ & unknown peaks (3) & Benzoic acid derivative $^{2}$ \\
\hline \multirow[t]{2}{*}{ unknown peaks $(3+\mathbf{1})$} & & glycerol-2-phosphate $^{2}$ \\
\hline & $\mathrm{J}$ & $\mathrm{N}$-acetylproline ${ }^{2}$ \\
\hline $\mathrm{E}$ & 1,5-dihydroxypentane ${ }^{2}$ & phosphate derivative $^{2}(2)$ \\
\hline 2,4,5-trihydroxypentanoic acid ${ }^{2}$ & 5-aminovaleric acid $^{2}(2)$ & xylitol $^{1}$ \\
\hline 2,4-dihydroxybutanoic acid ${ }^{2}$ & 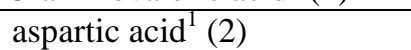 & pyroglutamic acid / glutamic acid $^{1}$ \\
\hline 4-hydroxyphenyllactic acid $^{2}$ & 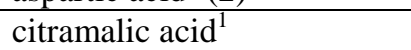 & sugar phosphate $^{2}$ \\
\hline 9-tetradecenoic acid ${ }^{2}$ & ethanolamine $^{2}$ & unknown peaks (8) \\
\hline benzaldehyde ${ }^{2}$ & formic acid $^{2}$ & \\
\hline${\text { decanoic } \text { acid }^{1}}$ & glutamic acid $^{1}(2)$ & $\mathrm{O}$ \\
\hline lactic acid $^{1}$ & hydroxylamine $^{2}$ & (benzene based) $^{2}$ \\
\hline levulinic acid $^{2}$ & $\mathrm{~N}$-acetylaspartic $\operatorname{acid}^{1}(2)$ & 1,2,3-trihydroxybenzoic acid ${ }^{2}$ \\
\hline${\text { octanoic } \text { acid }^{1}}^{2}$ & uracil $^{1}$ & 1,3-propanediol ${ }^{1}$ \\
\hline sugar phosphate $^{2}$ & N-acetyltryptophan ${ }^{1}$ & 1,6-anhydroglucose ${ }^{1}$ \\
\hline unknown peaks (7) & nonanoic $\operatorname{acid}^{2}$ & 1-cyclopropene(2-hydroxypropyl) ${ }^{2}$ \\
\hline
\end{tabular}


Supplementary Table 2 Continued

\begin{tabular}{|c|c|c|}
\hline Continued $\mathrm{O}$ & Continued $\mathrm{O}$ & $\mathrm{T}$ \\
\hline 2-hydroxyglutaric acid ${ }^{1}$ & phosphate derivative $^{2}$ & ethylene glycol ${ }^{2}$ \\
\hline 2-isopropylmalic acid ${ }^{1}$ & phosphate $^{1}$ & 2,4-imidazolidendione derivative ${ }^{2}$ \\
\hline 2-propanol,1,3-dibromo ${ }^{2}$ & phosphate $^{1}$ & sugar or sugar alcohol ${ }^{2}(2)$ \\
\hline 3-methyl, 1-2 dihydroxybutane ${ }^{2}$ & phosphate $^{1}$ & phosphate derivative $^{2}$ \\
\hline 3-methyl, 2-oxobutanoic acid ${ }^{2}$ & propanetriol or caffeic acid ${ }^{2}$ & unknown peak \\
\hline 4-hydroxybenzoic acid ${ }^{1}$ & putrescine $^{1}$ & \\
\hline 4-hydroxybutyric acid ${ }^{1}$ & succinic $\operatorname{acid}^{1}(2)$ & $\mathrm{U}$ \\
\hline 9-tetradecenoic acid ${ }^{2}$ & sucrose $^{1}$ & 2,3-butanediol ${ }^{1}$ \\
\hline acetic acid ${ }^{2}$ & sugar or sugar alcohol ${ }^{2}(12)$ & 4-hydroxyproline ${ }^{1}$ \\
\hline alpha-hydroxyisovaleric acid ${ }^{2}$ & sugar phosphate $^{2}(2)$ & 5-aminomethyl isoxazol-3-ol ${ }^{2}$ \\
\hline ammonium $^{1}$ & threitol or erythritol $^{1}$ & aminomalonic acid $^{2}$ \\
\hline citric acid $^{1}$ & ${\text { threonic } \text { acid }^{1}}^{1}$ & ammonium $^{1}$ \\
\hline dodecanoic acid $^{2}$ & triethanolamine $^{2}$ & mercaptoacetic acid $^{2}$ \\
\hline 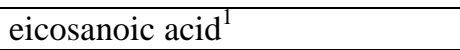 & trimethylamine-N-oxide ${ }^{2}$ & unknown peaks $(6)$ \\
\hline fatty $\operatorname{acid}^{2}(2)$ & unknown peak (33) & \\
\hline \multicolumn{3}{|l|}{ fructose $^{1}$} \\
\hline fumaric acid $^{1}$ & $\mathrm{P}$ & \\
\hline $\operatorname{glucose}^{1}(2)$ & 2-chlorotriethanolamine ${ }^{2}$ & \\
\hline glyceric acid-3-phosphate ${ }^{2}$ & 2-methyl, 4-hydroxybutyric acid ${ }^{2}$ & \\
\hline glycerol $^{1}$ & 2-monostearin ${ }^{2}$ & \\
\hline glycerol-3-phosphate ${ }^{2}$ & glycine $^{1}$ & \\
\hline heptadecanoic acid ${ }^{2}$ & phosphoric acid, monomethyl ester ${ }^{2}$ & \\
\hline hexadecanoic acid $^{1}(2)$ & pyruvic acid ${ }^{1}$ & \\
\hline \multicolumn{3}{|l|}{ uridine $^{2}$} \\
\hline xylose $^{1}$ & Q & \\
\hline isomaltose $^{2}$ & heptadecanoic acid ${ }^{2}$ & \\
\hline maltose $^{1}$ & tagatose $^{1}$ & \\
\hline monostearin $^{2}$ & unknown peaks (3) & \\
\hline \multicolumn{3}{|l|}{ myo-inositol $^{1}$} \\
\hline $\mathrm{N}$-acetylserine ${ }^{2}$ & $\mathrm{R}$ & \\
\hline \multicolumn{3}{|l|}{ octadecanoic acid ${ }^{1}$} \\
\hline \multicolumn{3}{|l|}{ oleic acid $^{1}$} \\
\hline palmitin $^{1}$ & $\mathrm{~S}$ & \\
\hline pantothenic acid ${ }^{1}$ & unknown peak & \\
\hline pentadecanoic acid ${ }^{2}(2)$ & & \\
\hline
\end{tabular}

